\section{Entsorgung kommunaler Spielräume}

\author{
Die Regulierung der Hausmüllentsorgung hat sich in den letzten Jahren stark \\ verändert. Ein Ergebnis ist, daß die kommunalen Entsorgungsträger, die bisher \\ das System dominiert haben, in Schwierigkeiten geraten. Zugleich erweist sich \\ ihr Handlungsspielraum zunehmend als begrenzt.
}

$\mathrm{A}$

Von Lilo Fischer nfang der 70er Jahre hat der Bund seine Gesetzgebungskompetenz im Bereich der Abfallbeseitigung durchgesetzt. Seitdem müssen sich die entsorgungspflichtigen Körperschaften (i.d.R. die Kreise) den bundeseinheitlich geltenden Regelwerken anpassen. Für die Hausmüllentsorgung heute maßgeblich sind das seit 1996 gültige Kreislaufwirtschaftsund Abfallgesetz (Krw-/AbfG), die 1991 verabschiedete und 1998 novellierte Verpackungsverordnung (VerpVo), sowie die Technische Anleitung Siedlungsabfall (TASi, in Kraft seit 1993). Die hiermit verbundenen strukturellen Veränderungen lassen sich wie folgt zusammenfassen:

- Die TASi schreibt ab 2005 die thermische Vorbehandlung von $\mathrm{zu}$ deponierendem Abfall vor. Ferner gelten neue Deponiestandards und die Kompostierung ist vorgeschrieben.

- Mit der VerpVO hat sich ein zweigleisiges Entsorgungssystem herausgebildet, das den Verpackungsabfall (,Wertstoffe“) aus der traditionell öffentlichen Entsorgung ausschließt. Diese hat damit nur noch die Verantwortung für die Entsorgung des „Restmülls“ inne.

- Mit dem Krw-/AbfG gelten nur noch eingeschränkte Überlassungspflichten an öffentliche Anlagen für gewerbliche Abfälle. Diese Abfallströme werden damit zum Teil privatisiert.

Damit ist jetzt nur noch die teure Kompostierung und die hohen Umweltstandards unterliegende Restmüllentsorgung in öffentlichen Händen, während gemäß dem dualen System das lukrative Geschäft mit den Wertstoffen in Händen der privaten DSD AG ist. Das Wegbrechen der Gewerbeabfälle (um bis zu 70 Prozent) führt heute zu Überkapazitäten der öffentlichen Anlagen, da nun der „billigste“ Entsorgungsweg gewählt werden kann (z.B. als Brennstoffersatz in Zementfabriken). Da es nun nicht mehr wie bisher notwendig ist, auf die staatlich vorgehaltene saubere - und daher kostenintensive - Entsorgungsinfrastruktur zurückzugreifen, wird diese entwertet. Das Ergebnis sind stetig steigende öffentliche Abfallgebühren trotz sinkender Abfallmengen.

Das Nebeneinander von privater und öffentlicher Entsorgungsschiene führte z.B. dazu, daß Betreiber von Müllverbrennungsanlagen (MVA) gezwungen sind, (gekauftes) öl zuzufeuern, seitdem die Wertstoffe fehlen. Ihre Forderung, daß kleine, verschmutzte Plastikverpackungsabfälle (immerhin 61 Prozent der gesammelten Plastikverpackungen) mit dem Restmill zusammen entsorgt werden, fiihrte in der Diskussion um die Novellierung der VerpVO nicht zum Erfolg. Zugleich erfordert eine konsequente Umsetzung der (ökologisch fragwürdigen) TASi-Anforderungen sogar den Ausbau des Netzes von MVAs. Der zuvor vorhandene Gestaltungsspielraum auf lokaler Ebene, der gerade durch die breite Anwendung von Mediationsverfahren bei der Wahl des anzuwendenden Entsorgungsmixes auch zunehmend genutzt wurde, wird hierdurch zunehmend eingeschränkt.

\section{Das Aus für lokale Innovationen?}

Stets waren es die Wahrnehmung von Entsorgungsengpässen einerseits und die mangelnde Akzeptanz von Entsorgungsanlagen andererseits, die die Innovationskraft der entsorgungspflichtigen Körperschaften beflügelte, Anstrengungen in Richtung eines akzeptierteren Abfallmanagements $\mathrm{zu}$ unternehmen. So haben sie beispielsweise angesichts drohender Entsorgungsengpässe in den 80er Jahren damit begonnen, die Hausmüllströme an der Quelle zu trennen, und sie gelten als Vorreiter in der Kompostierung organischen Abfalls. Durch die an Striktheit zunehmenden bundeseinheitlichen Vorgaben wird der Blick auf solche regionale Gegebenheiten heute verstellt und die im Grundgesetz verankerte kommunale Selbstverwaltungsgarantie mehr und mehr ausgehebelt. Ein Beispiel bildet die Möglichkeit, Abfall vor Ort zu vermeiden. Zunächst war es Kassel, die als erste Stadt ihren Willen zur ernstgemeinten
Lenkung der Verpackungswahl dokumentierte, indem sie Verpackungssteuern auf Einweg im Falle eines Verzehrs vor Ort erhob. Viele Kommunen folgten dem Beispiel, sei es mit der (irrigen) Aussicht auf eine weitere Einnahmequelle, sei es um der nicht enden wollenden Kostenspirale etwas an gut gemeintem Willen entgegenzusetzen. Fakt ist, daß die Einwegverpackungssteuer einen enormen Lenkungseffekt hin zu Mehrweg verbuchen konnte. Im Zuge der Klage von McDonalds gegen die Erhebung dieser Steuern fällte das Bundesverfassungsgericht 1998 jedoch ein Urteil, das die kommunale Einwegsteuer vor allem mit der Begrïndung verbot, daß schon auf Bundesebene eine umfassende Regelung in diesem Bereich durch die Zielsetzungen der Verpackungsverordnung bestehe (1).

Ein Wettbewerb zwischen unterschiedlichen (lokalen) Ansätzen ist damit in all denjenigen Bereichen nicht mehr möglich, die schon „oben" geregelt sind. Dabei sind weniger die effektiven Ergebnisse des zentralen Ansatzes als vielmehr die hier gesetzten Ziele für die Eingrenzung des Gestaltungsspielraumes relevant. Was den entsorgungspflichtigen Körperschaften heute an Lenkungsinstrumenten bleibt, um Abfälle an der Quelle zu vermeiden, ist lediglich die Abfallgebühr, die wiederum bei angemessener Höhe Ausweichreaktionen hervorruft.

Mit dem Regierungswechsel werden - bisher jedoch nur programmatisch - zwei Eckpfeiler des bestehenden Systems, zum einen die zentral vorgeschriebene Müllverbrennung und zum anderen die Organisation des dualen Systems der Hausmüllentsorgung, in Frage gestellt. Überlagert wird dies perspektivisch von einer weiteren Delegation legislativer Kompetenzen an die europäische Umweltpolitik, z.B. im Rahmen einer konsequenteren Verankerung der Produktverantwortung (2).

\section{Anmerkungen}

(1) Im selben Urteil wurden Sonderabfallabgaben auf Lönderebene sogar für verfassungswidrig erklärt.

(2) Einen vertieften Einblick in die damit verbundenen Fragestellungen wird z.B. der Schwerpunkt der Septemberausgabe 4/99 von Ökologisches Wirtschaften geben.

\section{Die Autorin}

Lilo Fischer ist freie wissenschaftliche Mitarbeilerin am IÖW.

Kontakt: Freiligrathstr. 10, 10967 Berlin,

Tel. 030/ 6945245, Fax 030/6945147,

E-mail LiselotteF@aol.com 
(c) 20I0 Authors; licensee IÖW and oekom verlag. This is an article distributed under the terms of the Creative Commons Attribution Non-Commercial No Derivates License (http://creativecommons.org/licenses/by-nc-nd/3.o/), which permits unrestricted use, distribution, and reproduction in any medium, provided the original work is properly cited. 